\title{
CONCEPT OF ARMATURE REACTION IN DC MACHINES
}

\author{
Pratik D. Solanki ${ }^{1}$, Nirav J. Patel ${ }^{2}$, Nikunj J. Dhimmar ${ }^{3}$, Jay A. Patel ${ }^{4}$

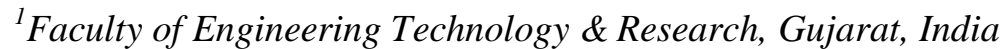 \\ ${ }^{2}$ Faculty of Engineering Technology \& Research, Gujarat, India \\ ${ }^{3}$ Faculty of Engineering Technology \& Research, Gujarat, India \\ ${ }^{4}$ CGPIT, Gujarat, India
}

\begin{abstract}
This paper gives the brief introduction about armature reaction. The problems such as high circulating current, poor commutation and sparking may occur at brush contact due to armature reaction. This paper also simplifies the logic related to armature reaction and gives the effective way to overcome the problem created due to armature reaction.
\end{abstract}

Keywords: Armature reaction, Commutation, GNA, Lorentz, MNA, Neutral zone

\section{INTRODUCTION}

In D.C machine shaft energy coming from mechanical domain goes into the magnetic domain, and then finally comes out to electrical domain. By means of the brushes and this comes out as the induced voltage. So, when applying a torque, loading on electrical side should reflect as load torque, such that the prime mover should supply extra torque to overcome that addition load. So it rotates with same rpm and as a result getting the same induced emf [1] [2] [3].

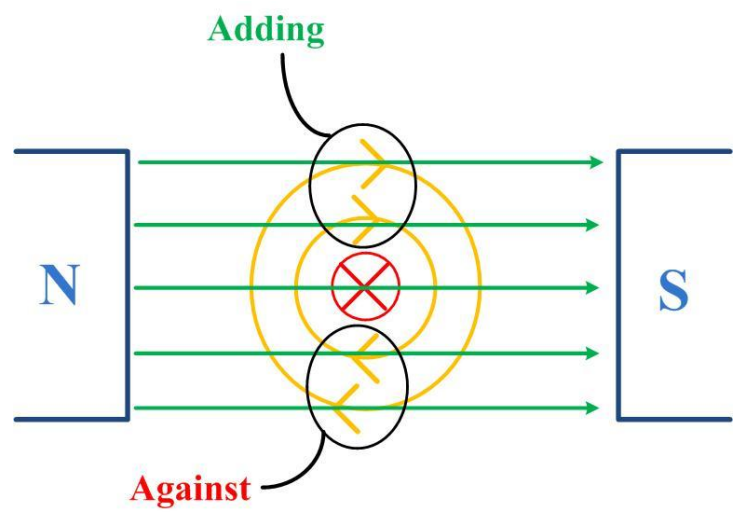

Fig 1 (a) Magnetic Flux lines of north-south pole,

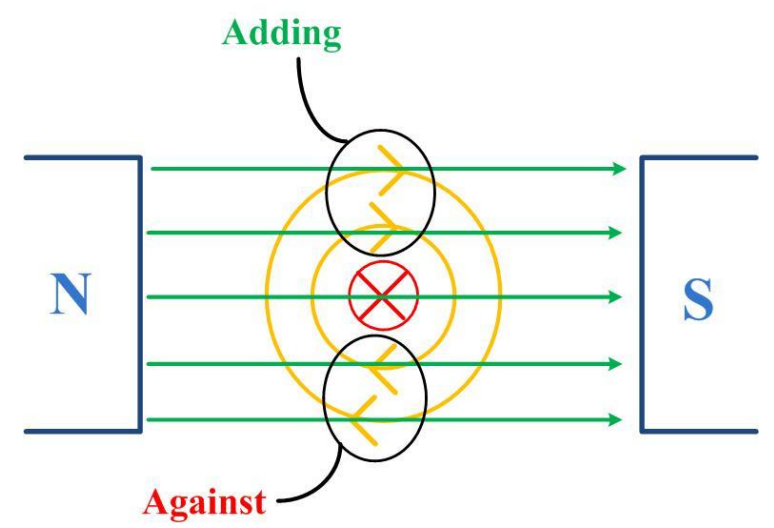

Fig 2 (b) Magnetic flux line due to current flowing from conductor
In the DC generator there is a North Pole and South Pole, flux line passing from North Pole to South Pole. Now, the conductor is placed in magnetic field and let the current flowing through it as shown in figure-1. Due to current the magnetic field is produced around the conductor. The field produced by conductor is against the main field at lower side of the conductor while it adding with the main field at upper side of the conductor.

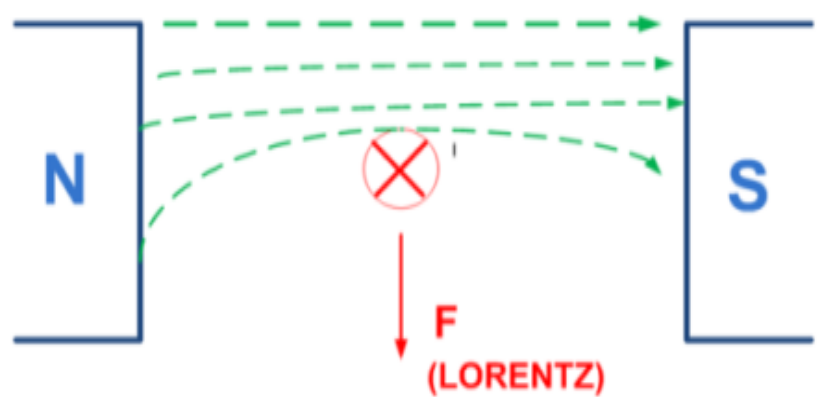

Fig 3 Lorentz force law

So the field on the bottom side of the conductor is tried to oppose the main field and field becomes weak while the upper field becomes stronger because of adding. Now upper field is acting like a rubber band, so it is trying to push the conductor in downward direction as shown in Fig.2.The force which pulls down the conductor is called Lorentz force which is given by,

$$
\mathrm{F}=\mathrm{BIL}
$$

Where, $\mathrm{B}=$ flux density in $\mathrm{Web} / \mathrm{m} 2$

$\mathrm{I}=$ current in $\mathrm{A}$

$\mathrm{L}=$ length of conductor in 


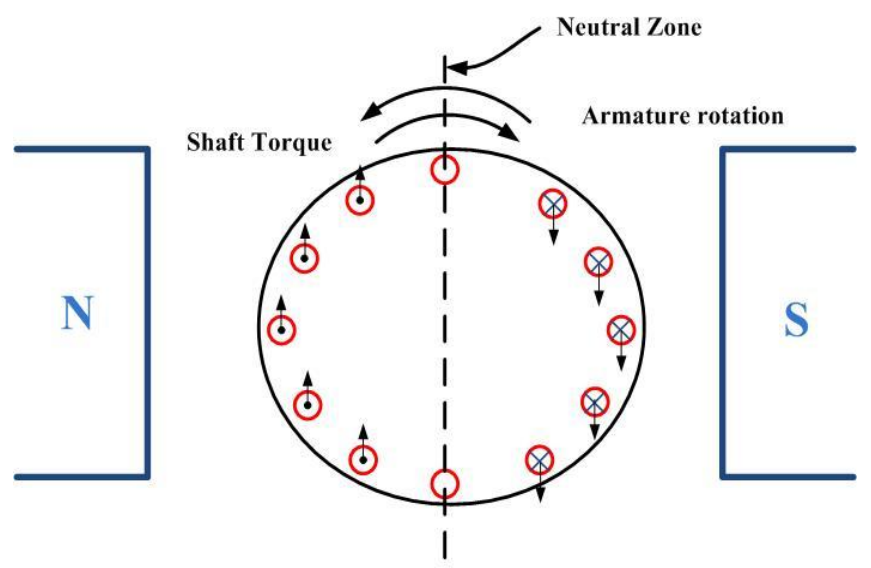

Fig 4 Forces produced by current flowing through the conductors in DC machine

\section{EFFECTS OF ARMATURE REACTION}

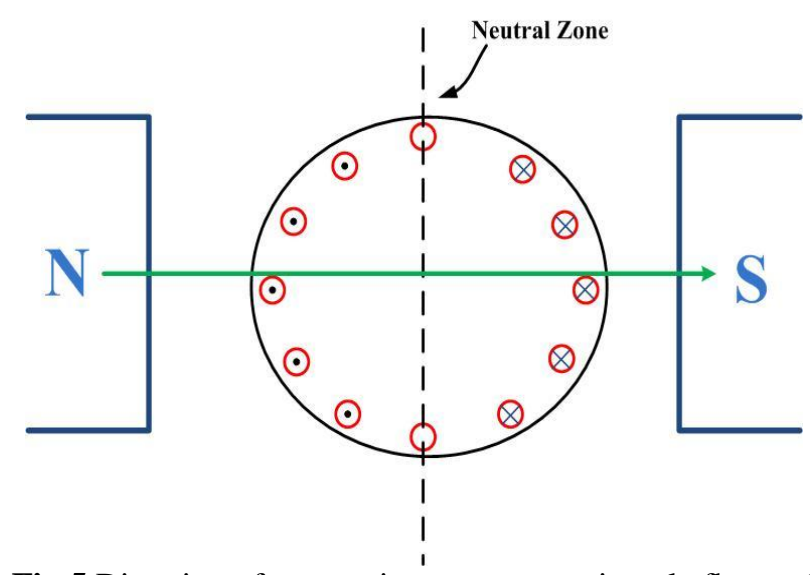

Fig 5 Direction of current in armature, main pole flux and neutral axis

Armature reaction is an undesirable effect that should be overcome in DC machine. In operation of DC Generator there is a flux between north-south poles and there is a coil in armature. Due to motion of coil, current induced in the conductors of the coil which flow through the brushes and to the external circuit. But, due to current flowing in the conductor of coil, there will be a field around it, now this field is going to interact with the main flux field of the north south magnetic poles which has been applied. Field created by conductor will distort the main field. Now in neutral zone which supposed to be as zero field zone where no induced voltage coil can exist, so no current flow though the armature conductors. Now let us look at the effect of this armature reaction. The north-south poles and conductors are in circular armature. The orthogonal plane is supposed to be the neutral zone where no induced voltage and thereby no induced current because at this point flux is equal to zero in that direction. The direction of flux flows is North Pole to South Pole. Now, there is a current in the previous loading effect diagram as shown in Fig. 4. Direction of load current flows through the conductor is shown in Fig. 4 in which current entering in the conductor and in another half portion of the armature is opposite from the previous one current is coming out of the conductor [3].

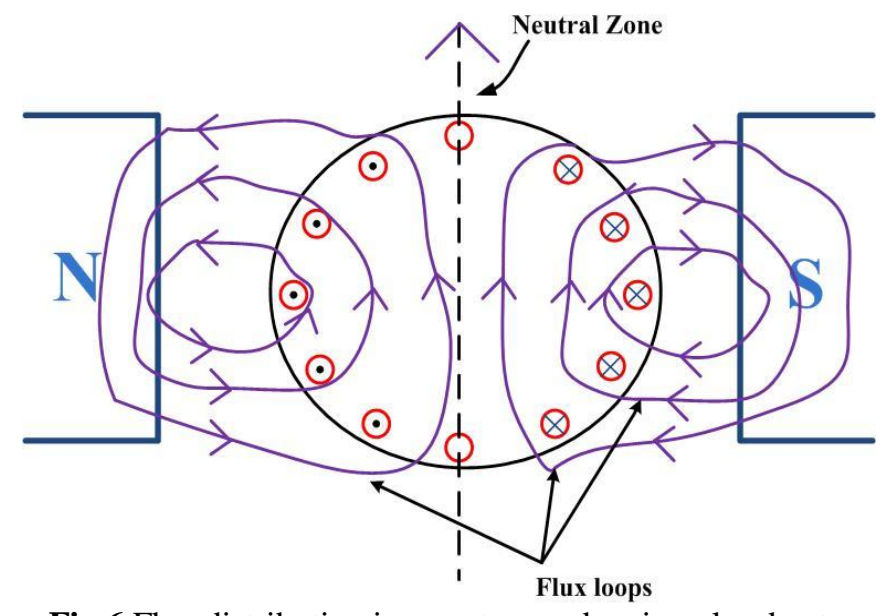

Fig 6 Flux distribution in armature and main poles due to current flowing in armature conductors

Now if, at the flux distribution produced by current flowing through conductors if armature coils, it will look like as shown in Fig. 5. On the left hand side of neutral zone, the flux is creating rings around conductors because there is current flowing within this loops and the direction is in clockwise which is given by the right hand rule. On another side also have a flux distribution or field distribution so a flux lines is in form of ring by the right hand rule and in anti-clockwise direction. These two fluxes are not going to contribute the neutral axis conductor placed at the centre. Now, inside the centre of armature of machine because of all the fluxes are pointing up, it means there is an effective flux which is pointing in upward direction. The flux is flowing in upward direction as shown in Fig. 5. So this leads to the following equivalent representation like north-south poles and the circular armature and was supposed to be a neutral zone where no flux [1] [3].

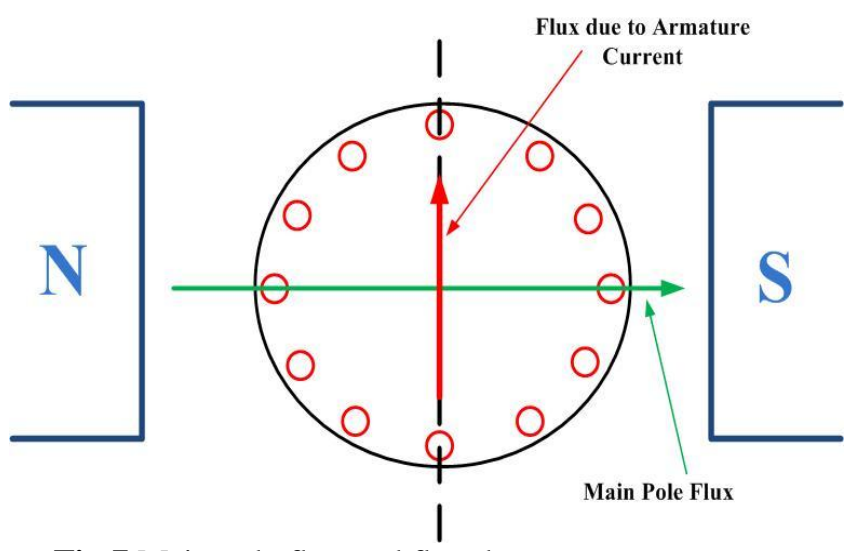

Fig 7 Main pole flux and flux due to armature current

There are two fluxes present in DC machine, the flux due to the north south-pole $(\phi)$ and another flux is in upward direction because all this add up inside the core this produce equivalent flux in this direction which is shown in Fig. 6. In the neutral zone there are not supposed to be any flux and therefore any coil will not have induced voltage but now due to the armature current which produces the loops which produces the flux due to the current flowing through the various conductors. 


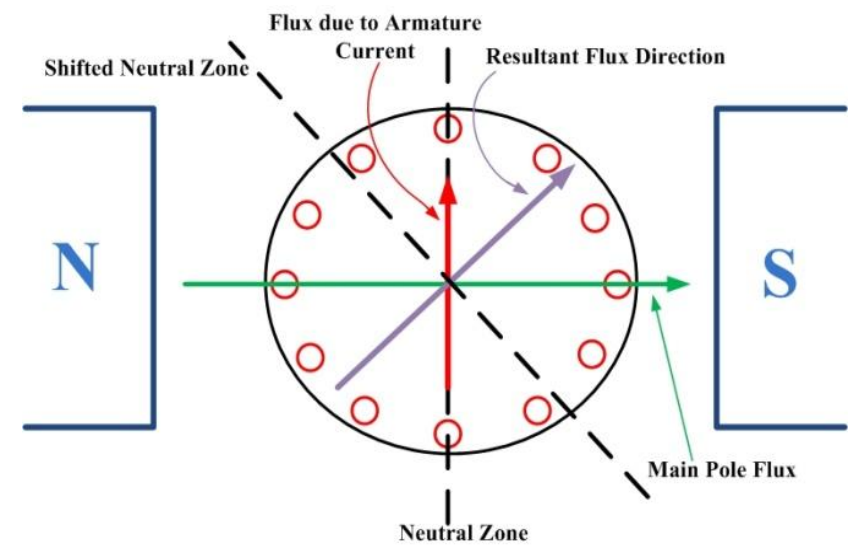

Fig 8 Resultant flux due to interaction between two fluxes

Now the flux created by armature current is in the upward direction and the direction of north-south pole flux is right angle to this flux. As shown in Fig. 7, the resultant flux is in between these two fluxes. So this would be the resultant flux direction. Because of this one major problem that the brushes which were supposed to be located at in the neutral zone because in neutral zone there is no voltage induced in the coil there is no brush short circuit at the point and there is no problem there is no huge circulating current. Now flux is produced due to the armature current and this flux always going to in this direction whatever may the position be of will resultant voltage being induced in the coil. Due to this armature current will short circuit the coil and produce huge short circuit circulating current and therefore I2R losses produced in armature winding and also results in sparking. Because when the brushes are passing over that coil there is a current flowing through that and there is an inductive reactance in the coil because of the coil is inductive in nature it is trying to suddenly break current in the coil, a huge spark results. So, due to these problems like circulating current which itself heat up the winding and the other one is sparking, so brushes should be place in neutral zone. So, the neutral zone can set at orthogonal to the main flux. Now a resultant flux is known and therefore orthogonal to the plane is this and these would be neutral zone which is shown in Fig. 7. The direction of rotation is consider as anti-clockwise. So neutral zone has shifted in the direction of rotation therefore brushes has been place in this new neutral zone. So at this new point it avoids the circulating current and also decreases the sparking.

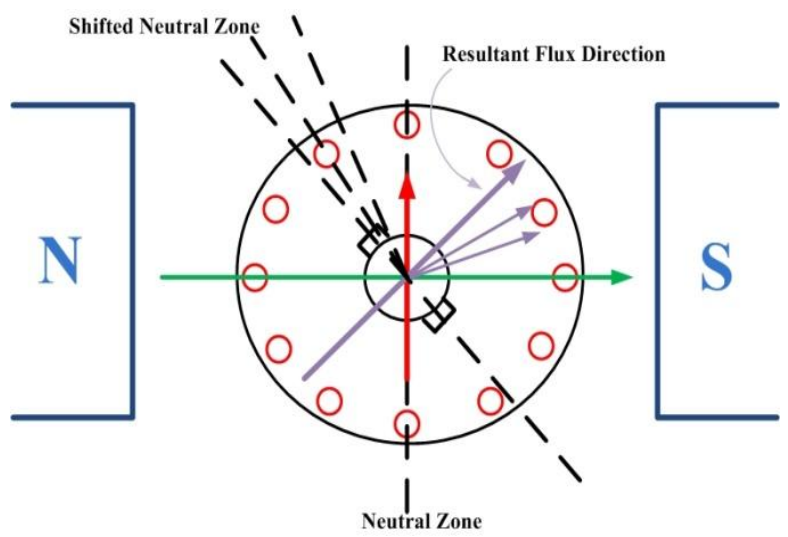

Fig 9 Variation in neutral axis due to vary the resultant flux
Now, by shifting the brushes like this, the problem would not have been simple resolved. Because the load current is varying quantity which means the flux due to armature current which indicating orthogonal to the north-south flux the amplitude keeps varying. So this amplitude keeps varying then the resultant could be in any direction depending upon the amount of load current as shown in Fig. 8. So orthogonal to that only will be the neutral zone. So, the neutral zone has much higher band of angle in which it can exist at various load current. Therefore the brushes cannot be dynamically positioned as the load current changes.

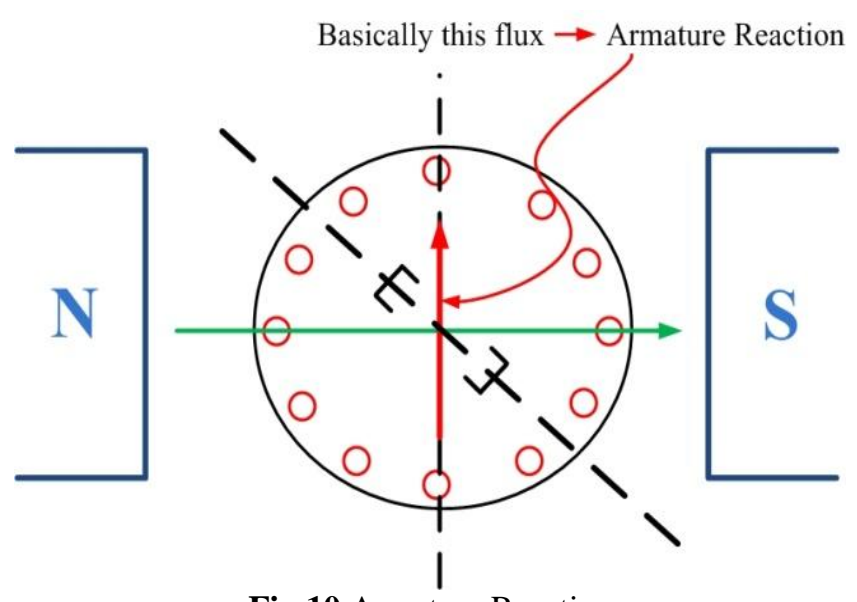

Fig 10 Armature Reaction

So therefore this flux which is created due to the load current of armature current flowing in and going to cause a problem. And this armature flux which is created due to the armature current flowing in the conductor is called as armature reaction which is shown in Fig. 9 [3] [4].

\section{SOLUTION OF ARMATURE REACTION}

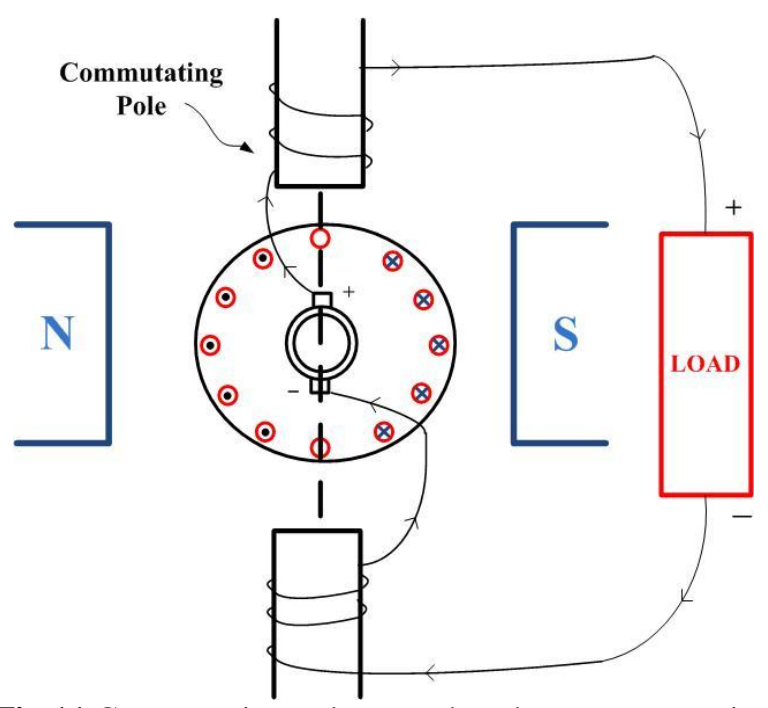

Fig 11 Commutating poles to reduced armature reaction

Let the commutator which is placed and also have the brushes in the neutral zone. Now two small poles are placed in between the north-south poles known as commutated poles. The positive terminal brush is connected to winding 
which is wound over this small pole and it connected to negative terminal as shown in Fig. 10. And the negative terminal brush is connected to winding which is wound over this small pole and it is connected to negative terminal. So, the current which flows from external load circuit is also flows from this commutated pole windings.

The $\phi$ is main flux of north-south pole which is orthogonal to neutral axis and $\phi r$ is the flux producing by armature reaction in whose direction is upward means orthogonal to main flux or in the direction of neutral axis as discuss above. Now when DC generator is loaded the current is flowing from the armature winding and it appears at brush terminals and current flows from commutating poles windings. The nature of the winding or the coil wound on the commutating poles and the currents flowing will produced the flux according to the right hand rule called commutating flux $\phi \mathrm{c}$ and the direction of flux is downward. So there are three fluxes $\phi, \phi r$ and $\phi c$ are presents in the DC machine shown in Fig. 11. Here direction of commutating flux $\phi c$ is opposite to the armature reaction flux $\phi r$.

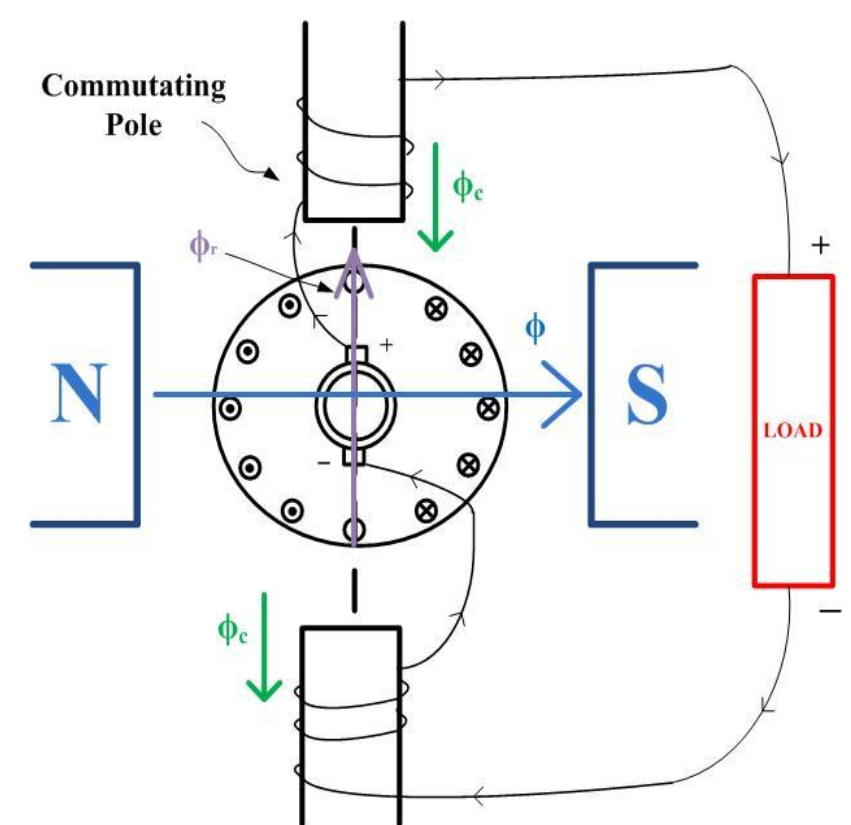

Fig 12 Commutating flux which oppose the armature flux to reduced armature reaction

So if the number of turns of winding on commutating poles is so match such that the flux $\phi c$ cancels out the flux due to armature reaction flux. Then the only flux that would exist is the north-south pole flux and this would still the orthogonal plane and would still into the neutral zone. This means the flux due to armature current or in fact the armature reaction is now zero. There by still maintaining the same neutral zone position and thereby do not shift position of the brush and the brushes can still stand same position and the voltages induced in the coil at the neutral zone to still the zero and therefore no short circulating current and therefore also no sparking or arcing when the commutator segment are passing over the brushes at that point that is the neutral zone [1] [3] [5].

\section{CONCLUSION}

There are many problems like high circulating current, poor commutation, and sparking may occur due to armature reaction which affects the performance of the machine. So in order to reduce the effect of armature reaction two small poles are placed in between the north-south poles are known as commutated poles and number of turns wound on commutator pole are so selected, the flux produced by the commutator pole will cancel out the flux which is due to armature reaction, so the effect of armature reaction is compensated

\section{REFERENCES}

[1] Stephen J. Chapman, "Electrical Machinery Fundamentals" Fourth Edition (McGraw-Hill: Elizabeth A. Jones, 2005).

[2] P. K. Mukherjee, S Chakravorti, "Electrical Machines" Second Revised Edition (Dhanpat Rai Publications: Ish Kapur, 2005).

[3] Theodore Wildi, "Electrical Machines, Drives and Power Systems" 6 Edition (Pearson, 2007).

[4] J.B.Gupta, "Theory and Performance of Electrical Machines" Fourteen Edition (S.K. Kataria \& Sons, 2009).

[5] A. E. Fitzgerald, Charles Kingsley, Jr. Stephen D. Umans, "DC Machines", in Sixth Edition, Electrical Machinery, (McGraw-Hill: Elizabeth A. Jones 2003) 367-370. 